\title{
ANP ROW SENSITIVITY AND THE RESULTING INFLUENCE ANALYSIS
}

\author{
William J. L. Adams \\ Decision Lens, Inc. \\ Arlington, VA USA \\ E-mail:wjadams@decisionlens.com
}

\begin{abstract}
Sensitivity analysis in the AHP settings gives useful and interesting information, especially when doing that analysis on the upper levels in the model. In the ANP setting (with feedback, and/or nodes having multiple destination clusters) the equivalent sensitivity on a particular judgment set yields either no sensitivity at all, or at the best, very minimal sensitivity. The other option of adjusting the global priorities in the ANP setting bypasses the ANP structure (i.e. the limit matrix calculation). This paper presents a method of ANP sensitivity calculations (ANP Row Sensitivity). That calculation reproduces the AHP calculation in that setting, gives useful sensitivity results, and preserves as much of the ANP structure as possible. With that defined, various influence analysis calculations are presented.
\end{abstract}

Keywords: anp, sensitivity, influence, super decisions, row sensitivity. 


\section{Introduction}

In AHP models sensitivity calculations allow one to perform two important analyses. First AHP sensitivity analyses can be used to do "what-if analysis" (e.g. what happens if we are not as tolerant of risks?). Secondly it can be used to perform various influence type of calculations (e.g. what criteria have the largest impact on the resulting alternative scores or rankings).

In ANP models both of these analyses would be insightful. However taking the standard AHP defined method of sensitivity analysis and translating to the ANP setting leads to essentially no sensitivity (when using the "with respect to node" method), or merely using global priorities analysis on multi-level models (Adams and Saaty, 1999 section 6 for details) which by-passes all of the limit matrix calculations, thereby skipping over the essential ANP structure.

This paper discusses ANP Row Sensitivity as defined in (Adams and Saaty 2013a) and implemented in Super Decisions. A sample set of calculations is performed on one of the standard models supplied with Super Decisions. Not only sensitivity calculations but also influence analysis is performed (which does several predetermined sensitivity changes and compares results before and after).

\section{Literature Review}

Super Decisions has had several types of sensitivity since its inception in the late 90's. The documentation for Super Decisions contains brief explanations of the calculations involved in section 6 (Adams and Saaty, 1999). However, the "with respect to node" calculation shows miniscule or non-existent sensitivity in networks with feedback (unless the sensitivity node happens to be an alternative, and in that case it is trivial linear sensitivity or a skewed curve because of the renormalization). Likewise the pairwise sensitivity is even less sensitive. The "global priorities" sensitivity is meaningless in a single level network setting and in a multi-level network setting only shows sensitivity on nodes with subnetworks and then only linear sensitivity (if one renormalizes by cluster instead of by network).

In (Adams and Saaty 2013a) a new sensitivity calculation is defined and described, called ANP Row Sensitivity. The main objective of this new calculation is to make ANP sensitivity calculations be a more direct counter-part to the AHP-Tree sensitivity calculation. The idea of ANP Row Sensitivity is to pick a node in a network and adjust its weight both globally and prior to the limit matrix calculation. This is accomplished by changing, not just the weight of the node with respect to a single node, but with respect to all nodes connecting to it. Of course, one cannot change the weights willy-nilly; rather, one has to preserve as much of the ANP structure as possible. With the assumption of preserving ANP structure there turns out to be essentially one way to parametrize the weight of the given node in the network (up to homeomorphism on the parameter). This new method of sensitivity simplifies down to the standard calculation for AHP-Trees and gives excellent sensitivity results both for single level and multi-level ANP networks. It also subsumes the previous "global priorities sensitivity" in Super Decisions into a framework that does sensitivity adjustments prior to limit matrix calculations (thus including the ANP structure within the sensitivity).

International Symposium of 2

the Analytic Hierarchy 
In addition, Super Decisions added influence analysis calculations based upon these sensitivity calculations. The purpose of the various influence analysis calculations is to systematically do what previously had been done with sensitivity using tedious "by hand" calculations, namely to find the most influential nodes within the model. There are several ways to define the most influential nodes. One way is to find the nodes that require the least change to induce a rank change in the alternatives (rank influence) described in (Adams and Saaty 2013c). Another way is to find the nodes where small changes give rise to the largest change in scores of the alternatives (marginal influence, i.e. a derivative calculation) described in (Adams and Saaty 2012b). Yet another method is to change the node weights, each in turn, by a fixed amount and calculate the change in alternative scores or rankings (called simply Influence in Super Decisions) described in (Adams and Saaty 2013b). Lastly one could allow the importance of the node in question approach 1.0 (i.e. it is the most important thing in the network) and see what the importance of the alternatives (or even the entire network structure) goes to (called perspective analysis in Super Decisions and described in (Adams and Saaty 2012a)).

\section{Hypotheses/Objectives}

This paper aims to describe how to use the new sensitivity calculations and influence analyses available in Super Decisions, and present sample calculations and analysis. With the work presented here one should have enough understanding of the calculations, software, and analyses to use Super Decisions to do the same on any given model. In a very real sense this paper is a companion document to (Adams and Saaty, 2012a,b and $2013 a, b, c)$ with more of an emphasis upon usage in Super Decisions and less emphasis upon the theory and full details of the calculation (which can be found in those documents).

\section{Research Design/Methodology}

The problem with sensitivity in the ANP setting prior to ANP Row Sensitivity calculations has been described already. ANP Row Sensitivity was designed to satisfy the following axioms.

1. Simplify to traditional AHP-Tree sensitivity when the given ANP network is a tree.

2. It needs to be based upon a single node in a network, and not a node and parent node combination (both because that is how it works in the tree setting and because choosing a node and parent node as the sensitivity location gives rise to little to no sensitivity).

3. It should preserve as much ANP structure as possible as the values are changed.

4. It should happen prior to limit matrix calculations, so that the sensitivity calculation actually reflects the ANP structure of the model (and not simply an after thought calculation like "global priority sensitivity").

The basic idea of ANP Row Sensitivity is to change an entire row of the super matrix (as opposed to the "with respect to" sensitivity which changes a single entry). The trick is to change the row in such a way as to preserve the ratios of entries as much as possible. The method is roughly to create a parameter $p$ that represents, in some way, the importance of the given node. As $p$ goes up, the node's importance goes up and as $p$ goes down the 
node's importance goes down (i.e. as $p$ goes up, so should the entries in the corresponding row of the super matrix, and similarly as $p$ goes down). In addition we want as $p$ approaches 1.0 the synthesized weight of the node should go to 1.0 and as $p$ goes to 0.0 the synthesized weight of the node goes to 0 . The latter is straightforward to accomplish and the former takes a bit of work, the details can be found in (Adams and Saaty 2013a).

Additionally the influence analysis calculations based upon ANP Row Sensitivity should allow one to determine the following.

1. The nodes most influenced by small changes. These are the nodes where uncertainty in pairwise comparisons would have the largest impact on the alternatives. This is called marginal influence in Super Decisions and described in (Adams and Saaty 2012b).

2. The nodes that cause rank changes the most quickly. This is called rank influence and is described in (Adams and Saaty 2013c).

3. The weights of the alternatives in the limit as this node become the most important thing in the network. This is the analogy of local synthesized weights for AHP models. It is called Perspective analysis in Super Decisions and is described in (Adams and Saaty 2012a).

4. Making a predetermined change to the weight of each node and calculating new alternative scores and then comparing those to the original, allowing one to calculate various distance metrics described in (Adams and Saaty 2013b).

\section{Data/Model Analysis}

The model used to present these calculations is the Hamburger_network sample model included with Super Decisions. This is a market share model determining the market shares of McDonalds, Burger King, and Wendy's that is a full ANP model with feedback and a single level. Prior to ANP Row Sensitivity, no real sensitivity or influence analysis calculations were possible with this model (that is, without subnetworks "global priorities" did not make sense, and "with respect to" sensitivity showed no sensitivity results).

\subsection{Node Sensitivity}

In Super Decisions, under the Computations menu choose Node Sensitivity and choose the 2 Taste node. The resulting graph looks like the following. 


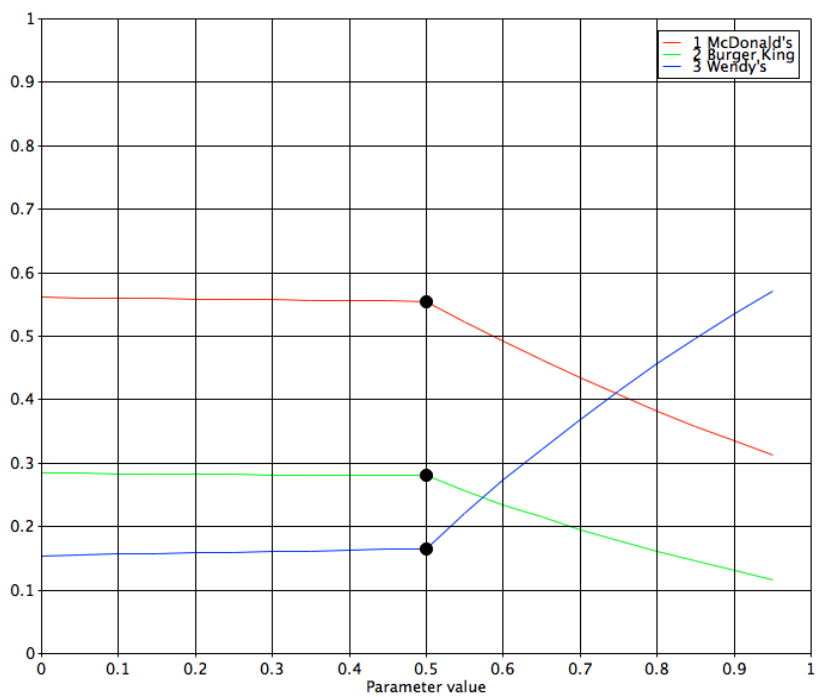

Notice the non-smooth point at $p=0.5$. This occurs because, by default $p=0.5$ is the original values, for $p$ above 0.5 the importance of the node goes up and below it goes down. In a network of any size there is always more room to go up than down, and so going upward results in more of a change. One can change the rest value for $p$ to a point that makes the graph smooth by choosing Computations-Influence/Sensitivity-OptionsSmart p0. Doing so results in the following graph (notice the dots have moved).

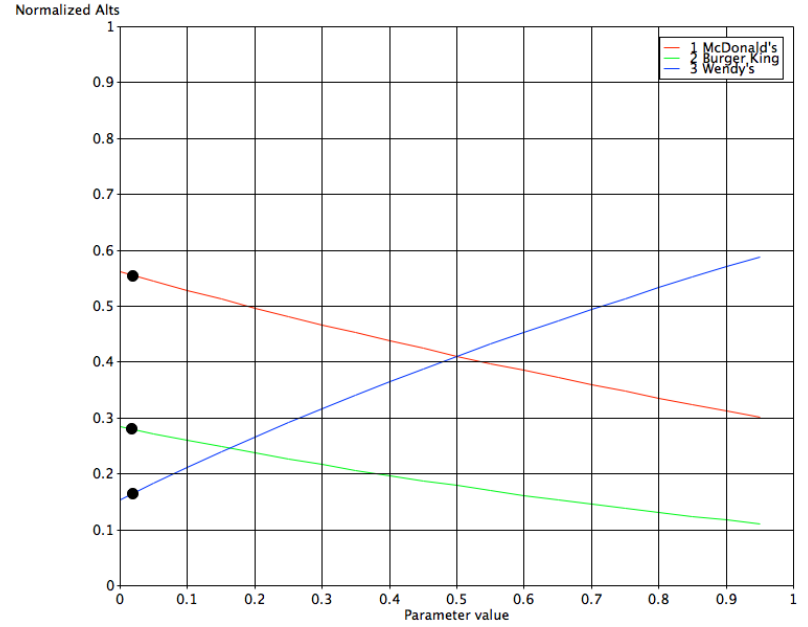

The locations of the dots represent the new resting locations for $p$. Notice that each graph could have a different resting $p$ value (or $p_{0}$ ). A key point to notice in both graphs is that once 2 Taste is important enough it causes Wendy's to beat Burger King, and then even beat McDonald's.

\subsection{Influence Analysis}

The next thing we will do is perform influence analysis on this model by first choosing $p_{0}=0.5$ again and then choosing Computations-Influence/sensitivity-Influence matrix which stores the result in a CSV file. The resulting data can be found in Table 1 in the appendix. The results show that the most influential nodes are the following (after removing the alternatives from that list).

1. 2 Taste: Brings up Wendy's and down the others.

International Symposium of 5

the Analytic Hierarchy

Washington, D. C. Process 
2. I Nutrition: Brings up Wendy's and down the others.

3. 3 Service: Brings up Wendy's and down the others.

4. 2 Promotion: Makes McDonald's even better.

\subsection{Rank Influence}

Rank influence calculates how much the importance of a given node must change to cause a change in the rankings of the alternatives (i.e. the change in the parameter $p$ that is needed to change the ranking of some alternatives). The smaller the change needed, the more rank influence that node has. To perform this calculation in Super Decisions choose Computations-Influence/sensitivity-Rank Influence matrix. The resulting data can be found in Table 2. The top influencers are the following.

1. 2 Taste:

2. 1 Nutrition:

3. 3 Service:

4. 3 Portion:

All cause Wendy's to increase and the others to decrease (with Wendy's changing ranking with Burger King). The only difference between this list and the last one is that Portion is the $4^{\text {th }}$ most rank influential whereas Promotion is the $4^{\text {th }}$ most influential in terms of raw changes.

\subsection{Marginal Influence}

Marginal influence calculates what the infinitesimal rate of change of the alternative scores are with respect to a given node (that is, the derivative of alternative score with respect to the parameter $p$ for a fixed node). This measures which nodes are most sensitive to small changes in priority (i.e. which nodes one must be very careful to have the best data possible about the priority of those nodes as they have the most immediate impact on alternative scores). To access this in Super Decisions choose ComputationsInfluence/sensitivity-Marginal Influence. The resulting data is in Table 3. The most marginally influential nodes are the following.

1. 1 Nutrition:

2. 2 Taste:

3. 3 Service:

4. 4 Cleanliness:

All of which raise the score Wendy's and drop the others (the derivative for Wendy's is positive for each and negative for McDonald's and Burger King). Note that 1 Nutrition is more marginally influential, but less rank influential and that 4 Cleanliness is the $4^{\text {th }}$ most marginally influential.

\subsection{Perspective Analysis}

Perspective analysis means, "what would the resulting alternative scores be if the given node was the most important" (this is accomplished by taking the limit as $p$ goes to 1.0 for the given node and then renormalizing the alternative scores). The results are in Tables 4 and 5. We see that the top four changers are the same as the regular influence analysis, i.e.

1. 2 Taste: Brings up Wendy's and down the others.

2. 1 Nutrition: Brings up Wendy's and down the others.

3. 3 Service: Brings up Wendy's and down the others. 
4. 2 Promotion: Makes McDonald's even better.

This is not particularly surprising as influence upper is setting $p$ to 0.9 (by default, this can be changed by choosing Influence Matrix (prompt for lower/upper)) and perspective is the result of the limit as $p$ goes to 1 . However from the table we can see, for instance that from the perspective of 2 Taste the scores of the alternatives should be

- McDonald's: 0.290

- Burger King: 0.104

- Wendy's: 0.606

That is, from the taste perspective Wendy's wins hands down (this is also the local priorities). For a case where the perspective and local priorities differ consider 3 Service. The following table shows the local alternative scores and perspective scores.

\begin{tabular}{|l|r|r|r|}
\hline 3 Service & McDonald's & Burger King & Wendy's \\
\hline Perspective & 0.374 & 0.153 & 0.473 \\
\hline Local & 0.332 & 0.139 & 0.529 \\
\hline
\end{tabular}

\subsection{Perspective Matrix Calculations}

The perspective matrix calculations are identical to the perspective analysis, but instead of just the alternative scores, we have the scores for all other nodes (so we can see how important all other nodes are relative to the given node). As we are setting $p$ towards 1 , we have to remove that score and renormalize so the diagonal is 0 . The only difference between GPerspective and BPerspective in Super Decisions is that GPerspective use the gnu multi-precision library (libgmp) to allow for arbitrary precision (which is a parameter to be set). In practice there is no calculated difference between these two, but to ensure no round off errors caused problems the arbitrary precision version was included.

\section{Limitations}

ANP Row Sensitivity and the associated influence analysis calculations are generalpurpose calculations that generalize AHP-Tree calculations and show good results. The only limitation is if one has an AHP model that is not a tree, ANP Row Sensitivity does not change a single weight with respect to its parent and thus that case cannot be handled (however the ANP Row Sensitivity would show information more in line with the tree counter part anyway).

\section{Conclusions}

ANP Row Sensitivity and influence analysis opens up a vista of analysis unavailable before. We can now see quickly which nodes are most influential (either through rank changes, raw changes, or infinitesimal changes).

One quick application of these results would be the following workflow.

1. Structure the model.

2. Quickly input scores without quibbling over small differences (a rough cut at priorities).

3. Do marginal influence analysis and discover which nodes are most influential.

4. Go back and do a more precise pairwise comparison set on those nodes (or more precise ratings) to make sure those numbers are exactly what they should be. 
In other words, we need not spend inordinate amounts of time on pairwise judgments for nodes that have no real impact.

Another usage is the Perspective analysis, to see what the alternative scores (or the entire network scores) are relative to a given node. This way we can understand better what is most important and least important from the vantage point of a given node.

Yet another usage is we can simply see which factors most impact the decisions overall; either, from the vantage point of rank change, or raw score changes, or other metrics (all of which are provided in the influence analysis results).

Lastly the ANP Row Sensitivity graphs with smart $p_{0}$ allow us to see quickly how changes to the importance of a given node affect the overall alternative scores whether we are in a single level network, or in multiple level networks.

\section{Key References}

Adams, W.J.L., Saaty, D.L. (2012a). US Patent No. 8,239,338. Washington DC: US Patent and Trademark Office.

Adams, W.J.L., Saaty, D.L. (2012b). US Patent No. 8,315,971. Washington DC: US Patent and Trademark Office.

Adams, W.J.L., Saaty, D.L. (2013a). US Patent No. 8,423,500. Washington DC: US Patent and Trademark Office.

Adams, W.J.L., Saaty, D.L. (2013b). US Patent No. 8,429,115. Washington DC: US Patent and Trademark Office.

Adams, W.J.L., Saaty, D.L. (2013c). US Patent No. 8,595,169. Washington DC: US Patent and Trademark Office.

Adams, W.J.L., \& Saaty, R. (1999). Super Decisions Software Guide, from http://www.ii.spb.ru/admin/docs/SuperDecisionsHelp2011.pdf. 
IJAHP Article: Adams, ANP ROW SENSITIVITY AND THE RESULTING INFLUENCE ANALYSIS

\section{Appendices}

Table 1: Influence Table

\begin{tabular}{|l|r|r|r|r|r|}
\hline $\begin{array}{l}\text { Net: Toplevel } \\
\text { network. }\end{array}$ & \multicolumn{1}{l|}{$\begin{array}{l}\text { Parameter } \\
\text { Value }\end{array}$} & \multicolumn{1}{l|}{ Distance } & McDonald's & \multicolumn{1}{l}{$\begin{array}{l}\text { Kurger } \\
\text { King }\end{array}$} & Wendy's \\
\hline Original Values & 0.5 & 0 & 0.554876 & 0.280166 & 0.164958 \\
\hline 1 Creativity:upper & 0.9 & 0.317555 & 0.62362 & 0.266903 & 0.112575 \\
\hline 2 Promotion:upper & 0.9 & 0.397404 & 0.692609 & 0.207929 & 0.099403 \\
\hline 3 Frequency:upper & 0.9 & 0.351269 & 0.672088 & 0.221055 & 0.107014 \\
\hline 1 Nutrition:upper & 0.9 & 2.411455 & 0.273236 & 0.164013 & 0.562748 \\
\hline 2 Taste:upper & 0.9 & 2.458792 & 0.31239 & 0.117058 & 0.570556 \\
\hline 3 Portion:upper & 0.9 & 0.496513 & 0.590802 & 0.162329 & 0.246862 \\
\hline 1 Price:upper & 0.9 & 0.224031 & 0.61304 & 0.256089 & 0.128003 \\
\hline 2 Location:upper & 0.9 & 0.392624 & 0.643282 & 0.257012 & 0.100192 \\
\hline 3 Service:upper & 0.9 & 1.65706 & 0.392761 & 0.168921 & 0.438304 \\
\hline 4 Speed:upper & 0.9 & 0.308791 & 0.54262 & 0.347326 & 0.114021 \\
\hline 5 Cleanliness:upper & 0.9 & 0.712685 & 0.436581 & 0.280868 & 0.282522 \\
\hline 6 Menu Item:upper & 0.9 & 0.813233 & 0.473608 & 0.227824 & 0.299108 \\
\hline 7 Take-out:upper & 0.9 & 0.034926 & 0.559987 & 0.283121 & 0.159197 \\
\hline 8 Reputation:upper & 0.9 & 0.219783 & 0.590572 & 0.21859 & 0.18592 \\
\hline 1 Creativity:lower & 0.1 & 0.002294 & 0.554653 & 0.280386 & 0.16458 \\
\hline 2 Promotion:lower & 0.1 & 0.003713 & 0.555129 & 0.27929 & 0.165571 \\
\hline 3 Frequency:lower & 0.1 & 0.113727 & 0.518527 & 0.297667 & 0.183719 \\
\hline 1 Nutrition:lower & 0.1 & 0.300644 & 0.520671 & 0.264775 & 0.214552 \\
\hline 2 Taste:lower & 0.1 & 0.282602 & 0.528213 & 0.260216 & 0.211576 \\
\hline 3 Portion:lower & 0.1 & 0.027369 & 0.558246 & 0.272498 & 0.169253 \\
\hline 1 Price:lower & 0.1 & 0.018794 & 0.555639 & 0.277768 & 0.161858 \\
\hline 2 Location:lower & 0.1 & 0.022143 & 0.559258 & 0.279418 & 0.161306 \\
\hline 3 Service:lower & 0.1 & 0.122203 & 0.54246 & 0.272413 & 0.185117 \\
\hline 4 Speed:lower & 0.1 & 0.040004 & 0.54998 & 0.281013 & 0.171557 \\
\hline 5 Cleanliness:lower & 0.1 & 0.049191 & 0.546883 & 0.280033 & 0.173073 \\
\hline 6 Menu Item:lower & 0.1 & 0.01491 & 0.556311 & 0.276275 & 0.167418 \\
\hline 7 Take-out:lower & 0.1 & 0.00236 & 0.553567 & 0.280076 & 0.164733 \\
\hline 8 Reputation:lower & 0.1 & 0.017402 & 0.556059 & 0.277056 & 0.162088 \\
\hline
\end{tabular}


IJAHP Article: Adams, ANP ROW SENSITIVITY AND THE RESULTING INFLUENCE ANALYSIS

Table 2: Rank Influence

\begin{tabular}{|l|r|r|r|r|r|r|}
\hline $\begin{array}{l}\text { Net: Toplevel } \\
\text { network. }\end{array}$ & \multicolumn{1}{l|}{$\begin{array}{l}\text { Parameter } \\
\text { Value }\end{array}$} & $\begin{array}{l}\text { Score } \\
\text { Score }\end{array}$ & \multicolumn{1}{l}{$\begin{array}{l}\text { McDonald } \\
\text { 's }\end{array}$} & \multicolumn{1}{l}{$\begin{array}{l}\text { lurger } \\
\text { King }\end{array}$} & Wendy's \\
\hline Original Values & 0.0 & 0.0 & 0.555 & 0.28 & 0.165 \\
\hline Creativity:upper & 0.99 & 0.0 & 0.0 & 0.632 & 0.26 & 0.108 \\
\hline Promotion:upper & 0.99 & 0.0 & 0.0 & 0.713 & 0.197 & 0.09 \\
\hline Frequency:upper & 0.99 & 0.0 & 0.0 & 0.699 & 0.209 & 0.092 \\
\hline Nutrition:upper & 0.576 & 0.846 & 0.846 & 0.494 & 0.253 & 0.253 \\
\hline Taste:upper & 0.574 & 0.849 & 0.849 & 0.508 & 0.246 & 0.246 \\
\hline Portion:upper & 0.786 & 0.416 & 0.416 & 0.58 & 0.21 & 0.21 \\
\hline Price:upper & 0.99 & 0.0 & 0.0 & 0.622 & 0.253 & 0.126 \\
\hline Location:upper & 0.99 & 0.0 & 0.0 & 0.651 & 0.252 & 0.097 \\
\hline Service:upper & 0.656 & 0.681 & 0.681 & 0.503 & 0.248 & 0.248 \\
\hline Speed:upper & 0.99 & 0.0 & 0.0 & 0.539 & 0.359 & 0.101 \\
\hline Cleanliness:upper & 0.939 & 0.104 & 0.104 & 0.438 & 0.281 & 0.281 \\
\hline Menu Item:upper & 0.789 & 0.411 & 0.411 & 0.511 & 0.245 & 0.245 \\
\hline Take-out:upper & 0.99 & 0.0 & 0.0 & 0.562 & 0.28 & 0.158 \\
\hline Reputation:upper & 0.99 & 0.0 & 0.0 & 0.598 & 0.212 & 0.19 \\
\hline Creativity:lower & 0.0 & 0.0 & 0.0 & 0.548 & 0.28 & 0.172 \\
\hline Promotion:lower & 0.0 & 0.0 & 0.0 & 0.544 & 0.284 & 0.172 \\
\hline Frequency:lower & 0.0 & 0.0 & 0.0 & 0.507 & 0.304 & 0.189 \\
\hline Nutrition:lower & 0.0 & 0.0 & 0.0 & 0.563 & 0.284 & 0.153 \\
\hline Taste:lower & 0.0 & 0.0 & 0.0 & 0.561 & 0.284 & 0.154 \\
\hline Portion:lower & 0.0 & 0.0 & 0.0 & 0.553 & 0.284 & 0.163 \\
\hline Price:lower & 0.0 & 0.0 & 0.0 & 0.556 & 0.277 & 0.167 \\
\hline Location:lower & 0.0 & 0.0 & 0.0 & 0.543 & 0.277 & 0.18 \\
\hline Service:lower & 0.0 & 0.0 & 0.0 & 0.558 & 0.282 & 0.16 \\
\hline Speed:lower & 0.0 & 0.0 & 0.0 & 0.549 & 0.275 & 0.176 \\
\hline Cleanliness:lower & 0.0 & 0.0 & 0.0 & 0.564 & 0.281 & 0.155 \\
\hline Menu Item:lower & 0.0 & 0.0 & 0.0 & 0.562 & 0.283 & 0.155 \\
\hline Take-out:lower & 0.0 & 0.0 & 0.0 & 0.555 & 0.28 & 0.165 \\
\hline Reputation:lower & 0.0 & 0.0 & 0.0 & 0.556 & 0.283 & 0.16 \\
\hline
\end{tabular}


IJAHP Article: Adams, ANP ROW SENSITIVITY AND THE RESULTING INFLUENCE ANALYSIS

Table 3: Marginal Influence

\begin{tabular}{|c|c|c|c|c|c|}
\hline $\begin{array}{l}\text { Net: Toplevel } \\
\text { network. }\end{array}$ & \begin{tabular}{|l|} 
D(Normal) \\
McDonald' \\
$\mathrm{S}$ \\
\end{tabular} & \begin{tabular}{|l|} 
D(Normal) \\
Burger \\
King \\
\end{tabular} & $\begin{array}{l}\text { D(Normal) } \\
\text { Wendy's }\end{array}$ & Total & Calc Err \\
\hline Original & 0.555 & 0.28 & 0.165 & 0.0 & 0.0 \\
\hline Creativity:upper & 0.127 & 0.003 & -0.13 & 0.182 & 0.0 \\
\hline Promotion:upper & 0.238 & -0.11 & -0.128 & 0.292 & 0.0 \\
\hline Frequency:upper & 0.205 & -0.111 & -0.095 & 0.252 & 0.0 \\
\hline Nutrition:upper & -0.854 & -0.393 & 1.247 & 1.561 & 0.001 \\
\hline Taste:upper & -0.661 & -0.484 & 1.145 & 1.408 & 0.001 \\
\hline Portion:upper & 0.096 & -0.216 & 0.119 & 0.265 & 0.0 \\
\hline Price:upper & 0.164 & -0.063 & -0.1 & 0.202 & 0.0 \\
\hline Location:upper & 0.266 & 0.033 & -0.299 & 0.401 & 0.001 \\
\hline Service:upper & -0.305 & -0.177 & 0.482 & 0.597 & 0.001 \\
\hline Speed:upper & -0.041 & 0.11 & -0.069 & 0.136 & 0.0 \\
\hline Cleanliness:upper & -0.318 & -0.011 & 0.329 & 0.458 & 0.001 \\
\hline Menu Item:upper & -0.109 & -0.129 & 0.238 & 0.291 & 0.0 \\
\hline Take-out:upper & -0.035 & 0.047 & -0.012 & 0.06 & 0.0 \\
\hline Reputation:upper & 0.09 & -0.121 & 0.032 & 0.154 & 0.0 \\
\hline Creativity:lower & 0.015 & -0.001 & -0.014 & 0.02 & 0.0 \\
\hline Promotion:lower & 0.026 & -0.01 & -0.016 & 0.032 & 0.0 \\
\hline Frequency:lower & 0.114 & -0.055 & -0.06 & 0.14 & 0.0 \\
\hline Nutrition:lower & -0.016 & -0.008 & 0.024 & 0.03 & 0.0 \\
\hline Taste:lower & -0.013 & -0.008 & 0.021 & 0.026 & 0.0 \\
\hline Portion:lower & 0.003 & -0.007 & 0.004 & 0.009 & 0.0 \\
\hline Price:lower & -0.003 & 0.007 & -0.004 & 0.009 & 0.0 \\
\hline Location:lower & 0.02 & 0.005 & -0.025 & 0.032 & 0.0 \\
\hline Service:lower & -0.007 & -0.003 & 0.01 & 0.012 & 0.0 \\
\hline Speed:lower & 0.012 & 0.01 & -0.022 & 0.027 & 0.0 \\
\hline Cleanliness:lower & -0.017 & -0.001 & 0.018 & 0.025 & 0.0 \\
\hline Menu Item:lower & -0.015 & -0.006 & 0.021 & 0.027 & 0.0 \\
\hline Take-out:lower & 0.0 & 0.0 & 0.001 & 0.001 & 0.0 \\
\hline Reputation:lower & -0.004 & -0.007 & 0.01 & 0.013 & 0.0 \\
\hline
\end{tabular}


IJAHP Article: Adams, ANP ROW SENSITIVITY AND THE RESULTING INFLUENCE ANALYSIS

Table 4: Perspective Unsorted

\begin{tabular}{|l|r|r|r|r|r|}
$\begin{array}{l}\text { Net: Toplevel } \\
\text { network. }\end{array}$ & \multicolumn{1}{l|}{$\begin{array}{l}\text { Parameter } \\
\text { Value }\end{array}$} & \multicolumn{1}{l|}{ Distance } & $\begin{array}{l}\text { Normal } \\
\text { McDonald's }\end{array}$ & $\begin{array}{l}\text { lormal } \\
\text { Burger } \\
\text { King }\end{array}$ & \multicolumn{1}{l|}{$\begin{array}{l}\text { Normal } \\
\text { Wendy's }\end{array}$} \\
\hline $\begin{array}{l}\text { Original } \\
\text { Values }\end{array}$ & 0.5 & 0.0 & 0.555 & 0.28 & 0.165 \\
\hline Creativity & 0.997 & 0.1 & 0.634 & 0.26 & 0.107 \\
\hline Promotion & 0.998 & 0.198 & 0.717 & 0.195 & 0.089 \\
\hline Frequency & 0.998 & 0.18 & 0.702 & 0.207 & 0.091 \\
\hline Nutrition & 0.999 & 0.542 & 0.249 & 0.156 & 0.595 \\
\hline Taste & 0.999 & 0.543 & 0.29 & 0.104 & 0.606 \\
\hline Portion & 0.998 & 0.169 & 0.594 & 0.146 & 0.26 \\
\hline Price & 0.994 & 0.082 & 0.622 & 0.252 & 0.126 \\
\hline Location & 0.997 & 0.123 & 0.653 & 0.251 & 0.096 \\
\hline Service & 0.999 & 0.379 & 0.374 & 0.153 & 0.473 \\
\hline Speed & 0.998 & 0.106 & 0.539 & 0.362 & 0.099 \\
\hline Cleanliness & 0.997 & 0.182 & 0.426 & 0.282 & 0.293 \\
\hline Menu Item & 0.998 & 0.192 & 0.457 & 0.223 & 0.32 \\
\hline Take-out & 0.988 & 0.01 & 0.562 & 0.28 & 0.158 \\
\hline Reputation & 0.997 & 0.086 & 0.599 & 0.211 & 0.191 \\
\hline
\end{tabular}

Table 5: Perspective Sorted

\begin{tabular}{|l|r|r|r|r|r|}
\hline $\begin{array}{l}\text { Net: Toplevel } \\
\text { network. }\end{array}$ & \multicolumn{1}{l|}{$\begin{array}{l}\text { Parameter } \\
\text { Value }\end{array}$} & \multicolumn{1}{l|}{ Distance } & McDonald's & \multicolumn{1}{l|}{$\begin{array}{l}\text { Kinger } \\
\text { Wendy's }\end{array}$} \\
\hline $\begin{array}{l}\text { Original } \\
\text { Values }\end{array}$ & 0.5 & 0.0 & 0.555 & 0.28 & 0.165 \\
\hline Taste & 0.999 & 0.543 & 0.29 & 0.104 & 0.606 \\
\hline Nutrition & 0.999 & 0.542 & 0.249 & 0.156 & 0.595 \\
\hline Service & 0.999 & 0.379 & 0.374 & 0.153 & 0.473 \\
\hline Promotion & 0.998 & 0.198 & 0.717 & 0.195 & 0.089 \\
\hline Menu Item & 0.998 & 0.192 & 0.457 & 0.223 & 0.32 \\
\hline Cleanliness & 0.997 & 0.182 & 0.426 & 0.282 & 0.293 \\
\hline Frequency & 0.998 & 0.18 & 0.702 & 0.207 & 0.091 \\
\hline Portion & 0.998 & 0.169 & 0.594 & 0.146 & 0.26 \\
\hline Location & 0.997 & 0.123 & 0.653 & 0.251 & 0.096 \\
\hline Speed & 0.998 & 0.106 & 0.539 & 0.362 & 0.099 \\
\hline Creativity & 0.997 & 0.1 & 0.634 & 0.26 & 0.107 \\
\hline Reputation & 0.997 & 0.086 & 0.599 & 0.211 & 0.191 \\
\hline Price & 0.994 & 0.082 & 0.622 & 0.252 & 0.126 \\
\hline Take-out & 0.988 & 0.01 & 0.562 & 0.28 & 0.158 \\
\hline
\end{tabular}

